\title{
An exact relation between willingness to pay and willingness to accept
}

\author{
Thomas A. Weber* \\ Department of Management Science and Engineering, Terman Engineering Center, Stanford University, Stanford, \\ CA 94305-4026, USA
}

Received 31 October 2002; accepted 19 February 2003

\begin{abstract}
I present a simple and precise relationship between the willingness-to-pay and the willingness-to-accept, or equivalently between the compensating and equivalent variations following an exogenous welfare change. One can be computed given the other as a function of income.
\end{abstract}

(C) 2003 Elsevier B.V. All rights reserved.

Keywords: Welfare change; Compensating variation; Equivalent variation

JEL classification: D11; D60

\section{Introduction}

Differences between willingness to pay (WTP) and willingness to accept (WTA) have been widely acknowledged in the empirical literature, where a persistent discrepancy in the two welfare measures is noted. ${ }^{1}$ On the normative side, progress has been made in bounding the difference between WTA and WTP. The equivalent and compensating variation (denoted by $E$ and $C$ ) are the welfare measures in standard demand theory (Hicks, 1939) that directly correspond to WTA and WTP. Willig (1976) noted that the difference between the two is likely to be small if the change in welfare is due to a price change of a market commodity. However, based on results by Randall and Stoll (1980), Hanemann (1991) shows that when the welfare change is induced by varying a nonmarket public good $q$, then differences between $C$ and $E$ can be arbitrarily large (infinite in the limit), depending on the degree of substitutability between $q$ and the other ordinary market commodities. He restates the bounds on the

\footnotetext{
*Fax: + 1-650-723-1614.

E-mail address: webert@stanford.edu (T.A. Weber).
}

${ }^{1}$ See e.g., Kahneman et al. (1991). 
difference obtained by Randall and Stoll in terms of elasticities, making plain the separate influence of substitution and income effect, each accounting for a portion of the deviation.

In this paper, I derive an explicit relation between $C$ and $E$ that holds for a large class of utility maximization problems. The idea is that, given a certain reference variation in the level of the nonmarket good, the induced equivalent variation $\hat{E}$ at an income level $\hat{y}=y-C$, reduced by the compensating variation, is equal to $C$. And this identity holds over the whole range of incomes, so that one welfare measure can be recovered from the other by the fundamental theorem of calculus. The obtained identity between compensating and equivalent variation allows bounding the difference, $E-C=$ WTA - WTP, if limits on the changes of one welfare measure are available, for instance through direct computation or observation.

\section{Preliminaries}

Suppose there are $n \geq 1$ conventional market goods $x_{1}, \ldots, x_{n}$ and one nonmarket good $q$. Let the consumer's preferences over the consumption of these goods be strictly convex, and represented by the increasing and strictly quasiconcave ${ }^{2}$ utility function, $u: \mathbb{R}^{n+1} \rightarrow \mathbb{R}$. In addition, to simplify the ensuing analysis, assume that $u=u(\mathbf{x}, q)$ is sufficiently smooth. Given a vector $\mathbf{p} \gg 0$ whose components represent prices for the respective market commodities, the consumer tries to find the optimal Hicksian commodity bundle $\mathbf{x}^{*}$ in $\mathbb{R}_{+}^{n}$, subject to her finite income $y \geq 0$. The classical utility maximization problem is given by

$$
\max _{x \geq 0} u(\mathbf{x}, q), \quad \text { subject to } \quad \mathbf{p} \cdot \mathbf{x}=y .
$$

Because of the continuity of $u$ there exists an optimal solution, $\mathbf{x}^{*}$, to the utility maximization problem Eq. (1), the components of which are described by the following Hicksian demand functions

$$
x_{i}^{*}=h^{i}(\mathbf{p}, q, y), \quad i=1, \ldots, n .
$$

The resulting indirect utility is defined as $v(\mathbf{p}, q, y)=u(h(\mathbf{p}, q, y), q)$. Note that the indirect utility is strictly increasing in income, so that for any $(\mathbf{p}, q) \gg 0,{ }^{3}$

$$
y^{0}<y^{1} \Rightarrow v\left(\mathbf{p}, q, y^{0}\right)<v\left(\mathbf{p}, q, y^{1}\right) \text {. }
$$

To value a change in the provision of the nonmarket $\operatorname{good} q$ from $q^{0}$ to $q^{1}$ we define the compensating variation $C(\mathbf{p}, y)$ and equivalent variation $E(\mathbf{p}, y)$ by

$$
\begin{aligned}
& v\left(\mathbf{p}, q^{1}, y-C(\mathbf{p}, y)\right)=v\left(\mathbf{p}, q^{0}, y\right), \\
& v\left(\mathbf{p}, q^{1}, y\right)=v\left(\mathbf{p}, q^{0}, y+E(\mathbf{p}, y)\right) .
\end{aligned}
$$

\footnotetext{
${ }^{2}$ Strict quasiconcavity is required only with respect to the conventional market goods $\mathbf{x}$ to guarantee the existence of a unique solution to the utility maximization problem (1).

${ }^{3}$ This result holds under weaker conditions on $u$ (only continuity and local nonsatiation of the underlying preferences are required, cf. Mas-Colell et al. (1995, p. 56)).
} 
Without loss of generality we assume $q^{1}>q^{0}$. The term $C(\mathbf{p}, y)$ is the income that a consumer would need to be compensated with in order to be indifferent between the higher level $q^{1}$ and the current level $q^{0}$. If the consumer is endowed with $q^{1}$, then $E(\mathbf{p}, y)$ is the income that yields equivalent utility to her as consuming at the lower level $q^{0}$. In other words, $C$ corresponds to the WTP, and $E$ to the WTA for the proposed welfare change.

Proposition 1. The willingness to pay, $C(\mathbf{p}, y) \in[0, y]$, and the willingness to accept, $E(\mathbf{p}, y) \in[0, \infty)$, for the welfare change from $q^{0}$ to $q^{1}$ exist, and are uniquely determined by Eqs. (3) and (4), respectively.

Proof. For any $(\mathbf{p}, q, y) \geq 0$ there is a unique $\mathbf{x}^{*}=h(\mathbf{p}, q, y)$ that solves the maximization problem Eq. (1). We obtain thus

$$
v\left(\mathbf{p}, q^{1}, y-0\right) \geq v\left(\mathbf{p}, q^{0}, y\right) \geq v\left(\mathbf{p}, q^{1}, y-y\right),
$$

so that by continuity of $v(\mathbf{p}, q, \cdot)$ there exists a $C(\mathbf{p}, y) \in[0, y]$ that solves Eq. (3). It is unique, since the LHS of Eq. (3) is strictly monotone in $C$. The existence of $E(\mathbf{p}, y)$ follows from a similar analysis of Eq. (4). Note first,

$$
\lim _{\lambda \rightarrow \infty} v\left(\mathbf{p}, q^{0}, y+\lambda\right) \geq v\left(\mathbf{p}, q^{1}, y\right) \geq v\left(\mathbf{p}, q^{0}, y+0\right) .
$$

Hence, a solution to Eq. (4) exists and must be unique by virtue of the strict monotonicity of $v\left(\mathbf{p}, q^{0}, y\right)$ in $y$.

\section{Main result}

Let $C(\mathbf{p}, y) \in[0, y]$ be the unique solution of Eq. (3) and set $\hat{y}=y-C(\mathbf{p}, y)$. Then the willingness to accept, $\hat{E}$, at the reduced budget $\hat{y} \leq y$ can be determined using Eq. (4),

$$
v\left(\mathbf{p}, q^{1}, \hat{y}\right)=v\left(\mathbf{p}, q^{0}, \hat{y}+\hat{E}\right) .
$$

Equivalently stated,

$$
v\left(\mathbf{p}, q^{1}, y-C(\mathbf{p}, y)\right)=v\left(\mathbf{p}, q^{0}, y-C(\mathbf{p}, y)+\hat{E}\right),
$$

so that Eq. (3), together with the injectivity of $v\left(\mathbf{p}, q^{0}, \cdot\right)\left(\right.$ since $\left.\partial_{y} v\left(\mathbf{p}, q^{0}, y\right)>0\right)$, implies $\hat{E}=C$. In other words $E(\mathbf{p}, y-C(\mathbf{p}, y))=C(\mathbf{p}, y)$ for all $y \geq 0$. Differentiating the last identity with respect to $y$, we obtain

$$
\partial_{y} E(\mathbf{p}, y-C(\mathbf{p}, y))=\frac{\partial_{y} C(\mathbf{p}, y)}{1-\partial_{y} C(\mathbf{p}, y)} .
$$

Note, by differentiating Eq. (3) with respect to $y$ and using the envelope theorem we find

$$
0<\partial_{y} v\left(\mathbf{p}, q^{0}, y\right)=\left(1-\partial_{y} C(\mathbf{p}, y)\right) \partial_{y} v\left(\mathbf{p}, q^{1}, y-C(\mathbf{p}, y)\right),
$$


so that $\partial_{y} C(\mathbf{p}, y)<1$ for all $y \geq 0$. Hence, the relation $\hat{y}=y-C(\mathbf{p}, y)$ is strictly monotonically increasing in $y$ and we define its inverse by $f(\mathbf{p}, \hat{y})=y$. Naturally $f(\mathbf{p}, \cdot)$ is continuous and strictly increasing and with Eq. (5) it is $\partial_{y} E(\mathbf{p}, \hat{y})=\partial_{y} C(\mathbf{p}, f(\mathbf{p}, \hat{y})) /\left(1-\partial_{y} C(\mathbf{p}, f(\mathbf{p}, \hat{y}))\right)$ for all $\hat{y} \geq 0$. Since $E(\mathbf{p}, y)$ can be written, using the fundamental theorem of calculus ${ }^{4}$ as

$$
E(\mathbf{p}, y)=E(\mathbf{p}, y-C(\mathbf{p}, y))+\int_{y-C(\mathbf{p}, y)}^{y} \partial_{y} E(\mathbf{p}, \xi) \mathrm{d} \xi
$$

we obtain the main result by a simple change of variables.

Theorem 1. Let $(\mathbf{p}, y) \geq 0$. The willingness to accept is related to the willingness to pay by

$$
E(\mathbf{p}, y)=C(\mathbf{p}, y)+\int_{y-C(\mathbf{p}, y)}^{y} \frac{\partial_{y} C(\mathbf{p}, f(\mathbf{p}, \xi))}{1-\partial_{y} C(\mathbf{p}, f(\mathbf{p}, \xi))} \mathrm{d} \xi
$$

where $f: \mathbb{R}_{+}^{n+1} \rightarrow \mathbb{R}_{+}$, with $f(\mathbf{p}, \xi)=\{y: \xi=y-C(\mathbf{p}, y)\}$, is a continuous single-valued function.

As a direct consequence of the first mean-value theorem in integral calculus we obtain the following corollary.

Corollary 1. There exists a constant $\mu(\mathbf{p}, y) \in[m, M]$, such that

$$
E(\mathbf{p}, y)=(1+\mu(\mathbf{p}, y)) C(\mathbf{p}, y),
$$

where $m=m(\mathbf{p}, y)$ and $M=M(\mathbf{p}, y)$ are the infimum and supremum of the set

$$
\mathscr{L}(\mathbf{p}, y)=\left\{\frac{\partial_{y} C(\mathbf{p}, f(\mathbf{p}, \xi))}{1-\partial_{y} C(\mathbf{p}, f(\mathbf{p}, \xi))}: \xi \in(y-C(\mathbf{p}, y), y)\right\}
$$

respectively.

Corollary 2. (i) If $E(\mathbf{p}, y)$ is monotonically increasing (decreasing) in $y$, then $E(\mathbf{p}, y) \geq(\leq) C(\mathbf{p}, y)$. (ii) If $C(\mathbf{p}, y)$ is increasing (decreasing) in $y$, then $C(\mathbf{p}, y) \leq(\geq) E(\mathbf{p}, y)$.

The dual relation to Eq. (6), formulated below, expressing $C(\mathbf{p}, y)$ as a function of $E(\mathbf{p}, y)$ can be obtained in a manner completely analogous to Theorem 1 .

Theorem 2. The willingness to pay is related to the willingness to accept by

$$
C(\mathbf{p}, y)=E(\mathbf{p}, y)-\int_{y}^{y+E(\mathbf{p}, y)} \frac{\partial_{y} E(\mathbf{p}, g(\mathbf{p}, \xi))}{1+\partial_{y} E(\mathbf{p}, g(\mathbf{p}, \xi))} \mathrm{d} \xi
$$

where $g: \mathbb{R}_{+}^{n+1} \rightarrow \mathbb{R}_{+}$, with $g(\mathbf{p}, \xi)=\{y: \xi=y+E(\mathbf{p}, y)\}$, is a continuous single-valued function.

\footnotetext{
${ }^{4}$ See Rudin (1976, p. 134).
} 


\section{Discussion}

The obtained relation between the equivalent and compensating variation directly relates WTA and WTP, so that from a normative viewpoint the question of which one is greater is resolved by determining the sign of the integral in either Eqs. (6) or (7). In addition, the difference between the two can be bounded, if the concrete problem at hand allows specifying limits on the slope of either $C$ or $E$. The exact relation can help avoid solving the dual problem to Eq. (1), when the sole objective is to obtain a bound on the difference or obtain one welfare measure from the other. Furthermore, Eqs. (6) and (7) may be useful as an alternative way of measuring the difference between WTA and WTP by estimating the slope of either compensating or equivalent variation over the relevant income interval.

\section{Acknowledgements}

This research was in part supported by the Risk Management and Decision Processes Center at the Wharton School of the University of Pennsylvania. I would like to thank Paul Kleindorfer and David Croson for helpful comments.

\section{References}

Hanemann, W.M., 1991. Willingness to pay and willingness to accept: How much can they differ? American Economic Review 81 (3), 635-647.

Hicks, J., 1939. Value and Capital. Clarendon Press, Oxford, UK.

Kahneman, D., Knetsch, J.L., Thaler, R.H., 1991. Anomalies: The endowment effect, loss aversion, and status quo bias. Journal of Economic Perspectives 5 (1), 193-206.

Mas-Colell, A., Whinston, M.D., Green, J.R., 1995. Microeconomic Theory. Oxford University Press, Oxford, UK.

Randall, A., Stoll, J.R., 1980. Consumer's surplus in the commodity space. American Economic Review 70 (3), $449-455$.

Rudin, W., 1976. Principles of Mathematical Analysis. McGraw-Hill, New York, NY.

Willig, R.D., 1976. Consumer's surplus without apology. American Economic Review 66 (4), 589-597. 